\title{
La microcirculación en el paciente crítico. Parte I: generalidades y fisiología en el paciente séptico
}

\author{
ALEJANDRO DONOSO F. ${ }^{1,2}$, DANIELA ARRIAGADA S. ${ }^{2}$, \\ PABLO CRUCES R. ${ }^{1}$, FRANCO DÍAZ R. ${ }^{1}$ \\ 1. Área de Cuidados Críticos. Unidad de Gestión Clínica de Niño. Hospital Padre Hurtado. \\ 2. Programa de Medicina Intensiva en Pediatría. Facultad de Medicina Clínica Alemana, Universidad del Desarrollo.
}

\begin{abstract}
Microcirculation in the critically ill. Part I: Review and physiology of septic patients

Severe sepsis and septic shock involve circulatory, inflammatory and metabolic disorders eventually resulting in a disruption of cellular energy. Microcirculatory disturbances are common in septic patients. Microcirculation is the primary site of oxygen and nutrients exchange to cells. Direct observation using Sidestream Dark Field (SDF) imaging has allowed direct visualization of microcirculatory failure in critically ill patients. Septic shock is characterized by weak or vulnerable microcirculatory units and heterogeneity of microcirculatory flow. Multiple mechanisms may contribute to these alterations, including endothelial dysfunction, altered glycocalyx, impaired inter-cell communication and adhesion and rolling of white blood cells and platelets. Many therapeutic interventions routinely used in the treatment of critically ill patients seem to result in limited changes in microcirculatory perfusion, irrespective of systemic hemodynamics, due to the heterogeneous nature of these changes and the potentially involved mechanisms. Therefore, microcirculatory alterations and their presence in states of shock, especially in septic shock, can represent diagnostic and severity stratification tools and may be a target for therapeutic intervention (microcirculatory resuscitation), besides suggesting a prognostic role.
\end{abstract}

(Key words: Microcirculation, endothelium, capillary, sepsis, shock, circulatory failure, organ failure).

Rev Chil Pediatr 2013; 84 (1): 83-92

\section{RESUMEN}

La sepsis grave y shock séptico involucran una compleja red de alteraciones circulatorias, inflamatorias y metabólicas que llevan finalmente a una disrupción energética celular. Las alteraciones microcirculatorias son frecuentes en el paciente séptico. La microcirculación corresponde al sitio anatómico-funcional donde ocurre el intercambio de oxígeno y nutrientes hacia la célula. Su observación directa mediante el uso de videomi-

Recibido el 26 de noviembre de 2011, devuelto para corregir el 16 de febrero de 2012, segunda versión el 10 de septiembre de 2012, aceptado para publicación el 5 de noviembre de 2012.

Este trabajo cumple con los requisitos sobre consentimiento/asentimiento informado, comité de ética, financiamiento, estudios animales y sobre la ausencia de conflictos de intereses según corresponda. 
croscopía como sidestream dark field (SDF) ha permitido la construcción de falla microcirculatoria como concepto clínico en el paciente crítico. En el shock séptico la existencia de unidades microcirculatorias débiles y la heterogeneidad del flujo microcirculatorio son características. Múltiples mecanismos contribuyen a estas alteraciones, incluyendo la disfunción endotelial, daño del glicocálix, alteración en la comunicación intercelular, adhesión y rotación de leucocitos y plaquetas. Diversas intervenciones terapéuticas empleadas rutinariamente en el tratamiento del paciente crítico parecen estar asociadas con cambios limitados en la perfusión de la microcirculación, con independencia de la hemodinamia sistémica, debido a la naturaleza heterogénea de estas alteraciones y los mecanismos potencialmente involucrados. Así, las alteraciones microcirculatorias y su persistencia en estados de shock, especialmente shock séptico, se pueden mostrar como una herramienta diagnóstica y de estratificación de gravedad, pudiendo ser un objetivo de intervención terapéutica (resucitación microcirculatoria) presentando a su vez un rol pronóstico.

(Palabras clave: Microcirculación, endotelio, capilar, sepsis, shock, falla circulatoria, falla orgánica).

Rev Chil Pediatr 2013; 84 (1): 83-92

\section{Introducción}

Uno de los principales objetivos de la monitorización hemodinámica en el paciente crítico es alertar al médico de la inminente insuficiencia cardiovascular antes que ocurra falla en tejidos u órganos ${ }^{1}$. Habitualmente la capacidad del sistema circulatorio es evaluada por parámetros clínicos y de laboratorio objetivables, por ejemplo frecuencia cardíaca, presión arterial, débito urinario, lactacidemia, exceso de base, etc. Sin embargo, los parámetros macrocirculatorios del sistema cardiovascular son actualmente considerados como marcadores poco fiables de perfusión tisular y en consecuencia inadecuados para estimar el estado hemodinámico global durante una condición crítica ${ }^{2}$.

El reconocimiento precoz de la inestabilidad hemodinámica en combinación con el entendimiento de la compleja fisiopatología es esencial. Por lo tanto, debemos monitorizar el parámetro correcto y secundariamente buscar el valor de meta adecuado.

La monitorización hemodinámica ideal debiese informarnos si las células están recibiendo suficiente oxígeno destinado a mantener la respiración mitocondrial para una adecuada producción de adenosín trifosfato (ATP) ${ }^{3}$. Hasta el momento no existe una herramienta de monitorización, disponible en tiempo real, para emplear a pie de cama que permita evaluar la entrega de oxígeno (Oxygen delivery, $\mathrm{DO}_{2}$ ).

En niños disponemos de una información escasa o limitada de la hemodinamia ya que no se mide débito cardíaco o saturación venosa mixta $\left(\mathrm{SvO}_{2}\right)$ en forma rutinaria, como si es frecuente de observar en adultos ${ }^{4-7}$.

La microcirculación juega un rol fundamental en el intercambio de gases y nutrientes, adaptándose en forma constante, mediante el control del tono vascular a las demandas metabólicas ${ }^{8}$. Así, la evaluación de la microcirculación puede ser una herramienta de valor adicional en la monitorización del niño críticamente enfermo.

En condiciones de normalidad la perfusión microvascular es controlada localmente, siendo el flujo sanguíneo tisular y la entrega de sustratos mantenidos a pesar de cambios en la presión arterial. El límite inferior de esta autorregulación de flujo varía entre pacientes, órganos, actividad metabólica y terapia vasoactiva asociada ${ }^{9}$. Sin embargo, el determinante primario del flujo sanguíneo a los órganos es la presión arterial, la hipotensión arterial es siempre patológica.

Una óptima macrocirculación es un prerrequisito para una adecuada perfusión microcirculatoria; no obstante, la restauración de la hemodinamia global no siempre significa que se ha logrado una adecuada perfusión regional, especialmente en condiciones de autorregulación alterada, tal como se observa en enfermedades críticas $(\text { shock })^{10}$. Las intervenciones terapéuticas destinadas a incrementar la perfusión de órganos deben estar acompañadas por una mejoría en la perfusión microvascular.

Las alteraciones en la perfusión microvascular, especialmente en etapa precoz del shock 
séptico, han sido implicadas en la falla orgánica y el Síndrome de disfunción orgánica múltiple $(\mathrm{SDOM})^{3,11-13}$.

Actualmente la evaluación de la microcirculación es posible con las nuevas técnicas imagenológicas disponibles ${ }^{14-16}$.

El objetivo de esta revisión es describir las características y el rol de las alteraciones microcirculatorias en el paciente críticamente enfermo.

\section{Shock e hipoxia celular}

El shock es una condición de naturaleza aguda, sindromática y patológica, donde existe un insuficiente entrega de oxígeno $\left(\mathrm{DO}_{2}\right)$ para cumplir las necesidades metabólicas tisulares, ocasionando un desbalance entre aporte y demanda el cual denominamos disoxia. Esta definición es operativa, pero presenta el inconveniente derivado la coexistencia de territorios con una buena relación entre perfusión y demanda de oxígeno, con otros donde no lo es, por los problemas de distribución del flujo en el shock séptico.

Recientemente se ha señalado que en la sepsis con una pobre respuesta terapéutica habitualmente encontramos variables hemodinámicas globales relativamente normales, pero con signos microcirculatorios asociados a mal pronóstico. Esta condición ha sido denominada síndrome de distress microcirculatorio y mitocondrial (SDMM) (figura 1), enfatizando la identificación del compartimento donde ocurre la falla y así explicar lo inadecuado de las estrategias terapéuticas en shock séptico que apuntan a corregir exclusivamente la hemodinamia sistémica. Por lo tanto, el shock séptico puede ser descrito fisiopatológicamente como un SDMM, donde tiempo y terapia forman parte integral de su definición. Debemos destacar que la condición de reversibilidad depende del tiempo transcurrido, gravedad

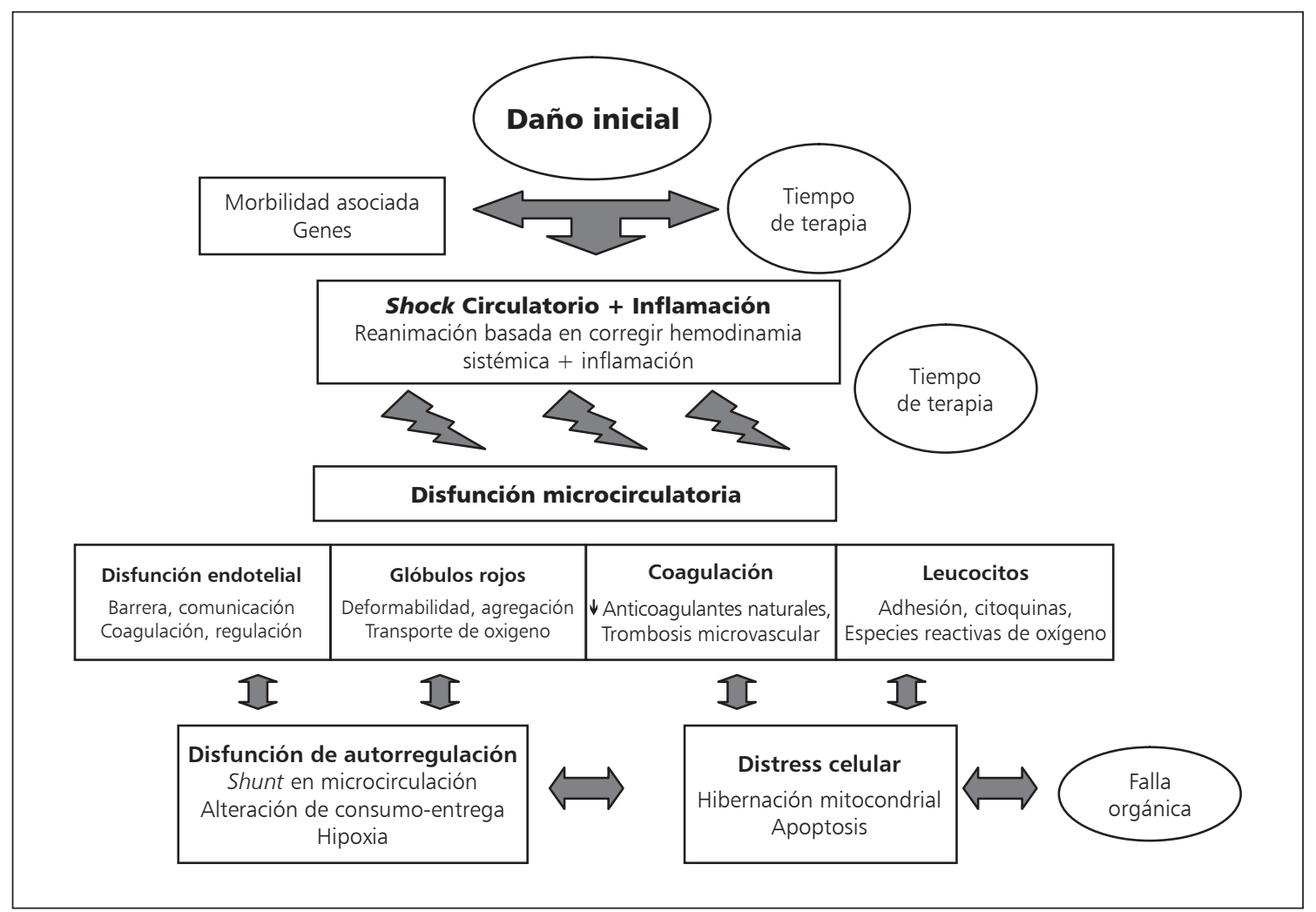

Figura 1. Síndrome de disfunción microcirculatoria y mitocondrial (SDMM). 
del daño hipóxico y de la magnitud del daño por reperfusión.

Su principal característica patogénica es el shunt en el transporte de oxígeno a los tejidos, ocasionado por la existencia de unidades microcirculatorias débiles (vide infra), lo cual resulta en disoxia regional.

En la actualidad podemos agrupar en tres los mecanismos que ocasionan hipoxia celular:

a) Falla macrocirculatoria: se evalúa frecuentemente en la práctica clínica mediante marcadores indirectos del flujo sanguíneo como lo son presión arterial media (PAM), gasto cardíaco y saturación venosa mixta $\left(\mathrm{SvO}_{2}\right)$.

b) Falla microcirculatoria: se manifiesta por una distribución anómala de flujo, con exclusión de arteriolas y capilares (shunt $)^{17}$. Se puede presentar en forma independiente del estado macrocirculatorio, siendo descrita frecuentemente como causa de hipoxia celular a pesar de la normalización de los parámetros hemodinámicos ${ }^{18}$. Esto reafirma la poca validez de las metas exclusivamente hidráulicas (PAM, PVC) en la reanimación del paciente séptico. Sin embargo, siempre debemos recordar que el primer objetivo es obtener un adecuado gasto cardíaco y presión arterial antes de tratar de manipular la microcirculación.

c) Falla mitocondrial o hipoxia citopática: se produce por desacoplamiento de los sistemas de producción energética celular (fosforilación oxidativa). Si su rol es patogénico o adaptativo es aún controversial.

\section{Fisiología de la microcirculación}

La microcirculación corresponde a los vasos menores de 100 micrones de diámetro, es decir a la red de arteriolas $(100-20 \mu \mathrm{m})$, capilares $(25-5 \mu \mathrm{m})$ y vénulas $(20-100 \mu \mathrm{m})$ que conectan el sistema arterial con el venoso.

Esta es ubicua y contiene el $90 \%$ del endotelio (la mayor superficie endotelial del cuerpo), siendo aquí donde ocurre el intercambio de oxígeno y nutrientes, remoción de $\mathrm{CO}_{2}$ y otros desechos metabólicos. Además, el endotelio presenta un papel clave en la inflamación y control de la coagulación.

La unidad microcirculatoria (UMC), según el modelo matemático conceptual del fi- siólogo A. Krogh ${ }^{19}$, corresponde al capilar y tejido que lo rodea, el cual es oxigenado por él (figura 2). Por ende, la cuantía de la densidad microvascular se relaciona con la distancia de difusión del oxígeno.

El flujo sanguíneo microcirculatorio normal es heterogéneo en tiempo y espacio, así durante condiciones de reposo aproximadamente un $30 \%$ de los capilares disponibles están perfundidos, no obstante, bajo condiciones de stress existe reclutamiento de capilares, lo que finalmente permite homogenizar la oxigenación de la microcirculación ${ }^{20}$.

Debemos recordar que una de las principales funciones microcirculatorias es la regulación del flujo sanguíneo (arteriolas de resistencia) con un importante descenso de la presión arterial desde la arteriola proximal a arteriola terminal (figura 3), permitiendo el ajuste fino de la distribución de la sangre a nivel de cada órgano, adaptándose a las demandas metabólicas locales de oxígeno.

Los capilares que se originan de las arteriolas terminales, están cubiertos por una delgada

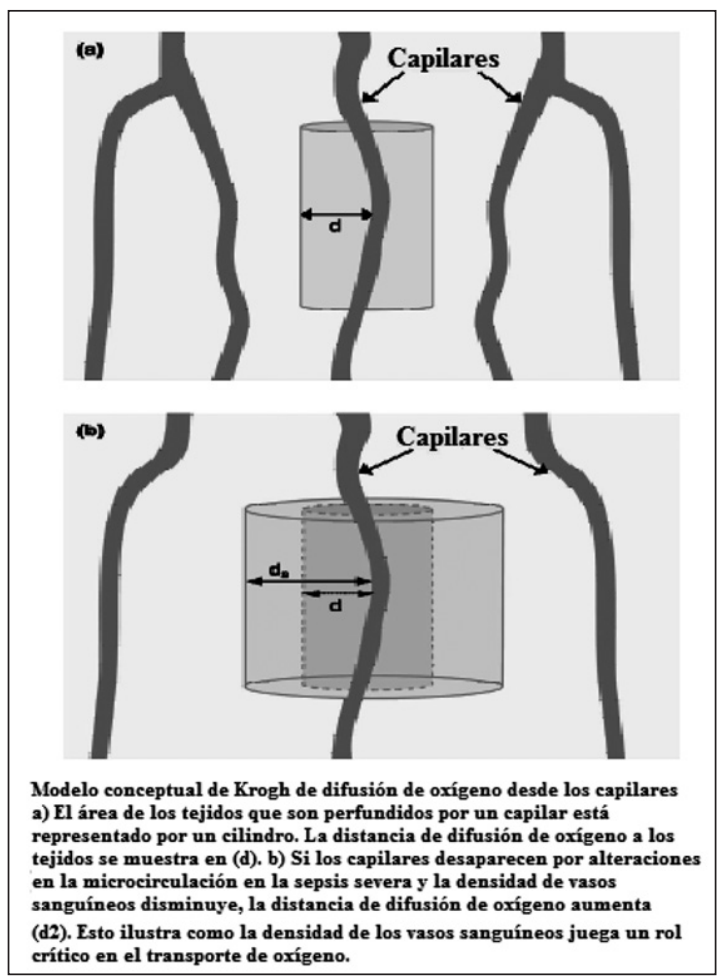

Figura 2. Unidad microcirculatoria. 


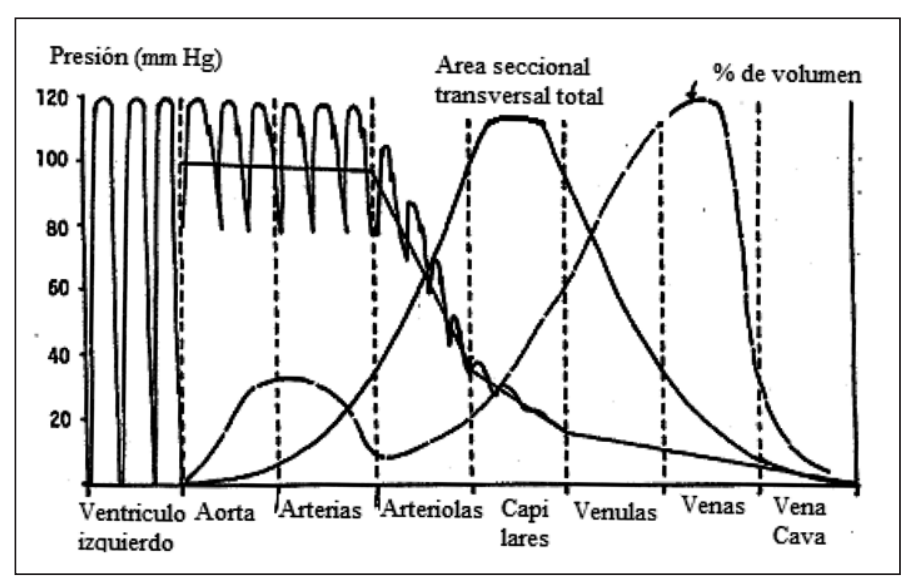

Figura 3. Gradientes de presión en la circulación sistémica, área de sección transversal total y distribución de la capacitancia vascular. superficie endotelial, siendo éstos los principales responsables del intercambio de oxígeno y nutrientes como también de la eliminación de productos del metabolismo.

Las redes microcirculatorias presentan una importante variación en relación a su morfología y densidad. La arquitectura varía entre diversos órganos siendo el tipo arborescente el más frecuente. El contacto entre vénulas, arteriolas y capilares es limitado, pero en algunos órganos como intestino, una disposición específica puede favorecer el mecanismo de intercambio por contracorriente.

La adhesión, rotación y migración leucocitaria, también como los cambios de permeabilidad ocurren principalmente en las vénulas, aunque esto puede ser variable entre diversos órganos.

Como se ha señalado previamente, las arteriolas son las encargadas del ajuste fino de la perfusión microcirculatoria, no obstante fenómenos que ocurren a nivel capilar o venular pueden afectar la perfusión, debido a la "conversación cruzada" (cross-talk) que ocurre entre las células endoteliales. Este mecanismo es logrado por un control electrofisiológico vía gap junctions (conexinas 40) 21,22. $^{2}$.

La sangre del territorio microcirculatorio es distinta a la arterial y se caracteriza por presentar un hematocrito menor (desciende hasta $50 \%$ del hematocrito sistémico) muy heterogéneamente distribuido (10-80\%). Esto es atribuido a un efecto del "vaso" y la "red". El primero se explica por el efecto Fahraeus-Linqdvist el cual provoca una reducción dinámica del hematocrito intravascular, debido a la migración axial de los eritrocitos hacia el centro del vaso, dejando el plasma cerca de su pared, lo que induce a diferencias en la velocidad eritrocitaria y plasmática. Derivado de ésto la diferencia de presión necesaria para mantener el flujo es menor.

El efecto ocasionado por la "red" es debido a que la distribución del hematocrito no es lineal en los puntos de ramificación vascular, encontrándose un menor hematocrito en vasos que presentan un menor ángulo. Además la $\mathrm{pO}_{2}$ es menor en la vecindad de la pared vascular, debido al consumo de oxígeno endotelial.

Todo lo anteriormente señalado ocasiona que la distribución del oxígeno sea muy heterogénea en la microcirculación, resultando de esta forma particularmente vulnerable a la hipoxia. Finalmente, el $\mathrm{DO}_{2}$ microcirculatorio no se puede predecir desde el $\mathrm{DO}_{2}$ global.

\section{Cambios post natales}

Debemos tener presente que en la primera semana de vida post natal el consumo de oxígeno $\left(\mathrm{VO}_{2}\right)$ aumenta entre dos a tres veces, ésto se debe a un incremento del trabajo respiratorio y a la mayor función gastrointestinal ocasionada al alimentarse; por otra parte los elevados niveles de hemoglobina fetal reducen la extracción de oxígeno. Estos factores son compensados por el mayor flujo sanguíneo microcirculatorio a esta edad.

Sin embargo, la densidad capilar funcional evaluada a nivel de la mucosa bucal, dismi- 
nuye después de la primera semana de vida neonatal. Estos cambios precoces parecen tener relación con la adaptación post natal más que con la edad post-concepción ${ }^{23}$. El modelo "tipo adulto" de microcirculación a nivel cutáneo es alcanzado a los tres meses de edad ${ }^{24}$. Un importante factor en este desarrollo es el enfriamiento local, no siendo claro si lo mismo se puede aplicar para otros lechos microvasculares que no están directamente expuestos a temperatura ambiental ${ }^{25}$.

\section{Mecanismos involucrados en la regulación de la perfusión microcirculatoria en condiciones de normalidad}

La perfusión de los tejidos, por ende el transporte de oxígeno, es logrado por dos mecanismos principales que son la difusión $\mathbf{y}$ convección ${ }^{26}$.

El componente difusivo está determinado por la diferencia entre la $\mathrm{pO}_{2}$ capilar $(\sim 30$ $\mathrm{mmHg}$ ) y mitocondrial ( $\sim 20 \mathrm{mmHg})$, un factor que es directamente proporcional a la distancia física entre el capilar y la célula (densidad vascular funcional). El segundo mecanismo que condiciona el transporte de oxígeno es el convectivo, definido como el número de células transportadoras de oxígeno por unidad de tiempo en un capilar dado (flujo).

En un escenario de normalidad fisiológica, la microcirculación es la responsable de la regulación fina de la perfusión para cubrir los requerimientos de oxígeno tisular, lo cual se logra reclutando y desreclutando capilares, es decir limitando el flujo en capilares que perfunden áreas con bajo requerimiento de oxígeno y aumentándolo en áreas con alto requerimiento. Este proceso implica un control local del flujo, donde juegan un papel la inervación simpática perivascular, la cual influye en el tono arteriolar, la comunicación retrógrada a lo largo del endotelio la que es mediada por las células endoteliales y el rol de los eritrocitos como sensores intravasculares ${ }^{27}$.

\section{¿Qué impulsa el flujo de sangre en los capilares?}

Según la ley de Poiseuille, el flujo en el capilar es proporcional a la diferencia de presión

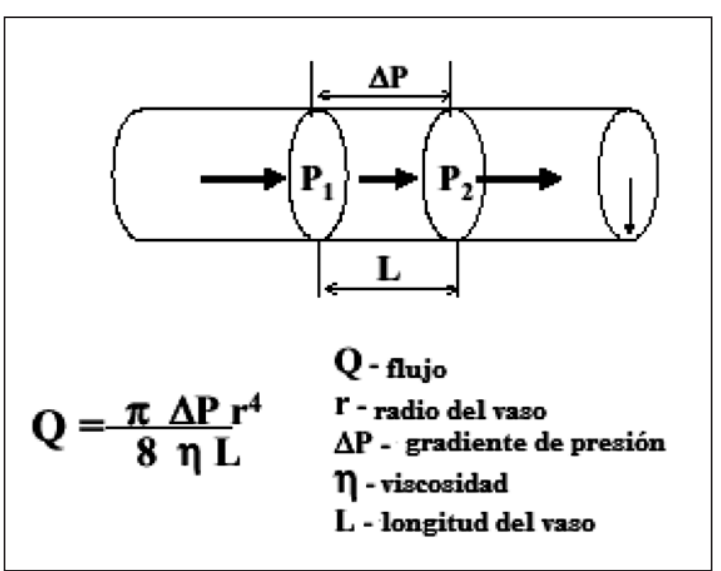

Figura 4. Ley de Poiseuille.

en los dos extremos del vaso (gradiente de presión, $\Delta \mathrm{P}$ ) y la cuarta potencia del radio capilar (r) e inversamente proporcional al largo capilar (L) y la viscosidad de la sangre (figura 4).

Como el largo del capilar y la viscosidad no pueden ser manipulados, el flujo capilar sólo puede ser modificado por dilatación local y/o incremento de la gradiente de presión (mediante vasodilatación de las arteriolas de resistencia).

Además, por presentar un hematocrito menor que el sistémico, por la presencia de la capa plasmática en la superficie endotelial, siendo en consecuencia el hematócrito proporcional al radio capilar, así ante una marcada vasodilatación aumentará la entrega de oxígeno local como resultado de la combinación del incremento del flujo y el contenido de oxígeno.

\section{Alteraciones microcirculatorias en la sepsis}

El intercambio de oxígeno depende de diversos factores tales como: contenido arterial de oxígeno, consumo de oxígeno, viscosidad sanguínea, flujo capilar y arteriolar, constante de difusión de los gases y la distancia de las células al vaso sanguíneo más cercano ${ }^{28}$.

Debido a ésto, la entrega tisular de oxígeno a nivel de la microcirculación no puede extrapolarse de la entrega de oxígeno sistémico, como tampoco del regional, siendo su distribución heterogénea y con un control predominante local del flujo microvascular, en búsque- 
da de un adecuado resguardo de las demandas tisulares.

En condiciones de normalidad los mecanismos de autorregulación sistémicos, regionales y locales, tanto metabólicos $\left(\mathrm{pH}, \mathrm{pCO}_{2}\right.$, $\mathrm{K}^{+}, \mathrm{O}_{2}$, oxido nítrico), miogénicos (fuerzas de tensión y elongación) como neurohormonales, aseguran una adecuada distribución del flujo para cubrir las necesidades tisulares. Sin embargo, en condiciones patológicas la pérdida de estos ocasiona disfunción microcirculatoria. Además los desórdenes que ocurren frecuentemente en el paciente crítico, como son desarrollo de inflamación, stress oxidativo, activación de la coagulación, disfunción endotelial, alteraciones reológicas y edema intersticial contribuyen a una alteración mayor del ambiente microcirculatorio.

En el shock séptico se "amputa" la microcirculación, observándose diversas alteraciones como una disminución de la densidad de capilares funcionales, un bajo flujo capilar (flujo ausente o intermitente), lo que determina un incremento de la heterogeneidad de la perfusión. Asimismo hay un aumento de la permeabilidad, todo lo cual finalmente dificulta la extracción de oxígeno (shunt funcional).

Estas alteraciones son ocasionadas por el rol de mediadores inflamatorios (factor de necrosis tumoral, tumoral necrosis factor, TNF; interleuquinas, IL). La endotelina, un potente vasoconstrictor endógeno, que se encuentra aumentada en la sepsis, puede alterar el flujo sanguíneo microcirculatorio como también este puede verse afectado por la liberación local de oxido nítrico, contribuyendo de este modo a un desbalance entre agentes vasodilatadores y vasoconstrictores.

Múltiples mecanismos pueden explicar las alteraciones microcirculatorias vistas en la sepsis grave y/o shock séptico. Estos son:

1. Disfunción endotelial (poca sensibilidad a agentes vasoconstrictores y vasodilatadores).

2. Expresión de moléculas de adhesión.

3. Alteración de la "conversación cruzada" entre células endoteliales por desacoplamiento de conexinas $^{29}$.

4. Alteración reológica de los hematíes, siendo más esféricos y con dificultad para modificar su forma, ocasionado por alteraciones en el contenido de acido siálico de la membrana eritrocitaria ${ }^{30}$.

5. Rotación plaquetaria y aumento de la agregación-adhesión de leucocitos a la célula endotelial, teniendo un rol significativo al ocasionar trombos obstructivos en el lumen de los microvasos. Sin embargo, se señala que la formación de microtrombos es infrecuente de observar en sepsis experimental ${ }^{31}$ (figura 5).

6. El papel del glicocálix, el cual es la cobertura de glicosaminoglicanos de la célula endotelial que facilita la circulación de los glóbulos rojos y limita la adhesión de leucocitos y plaquetas en los microvasos, no puede ser obviado, pues todas las células circulantes es-

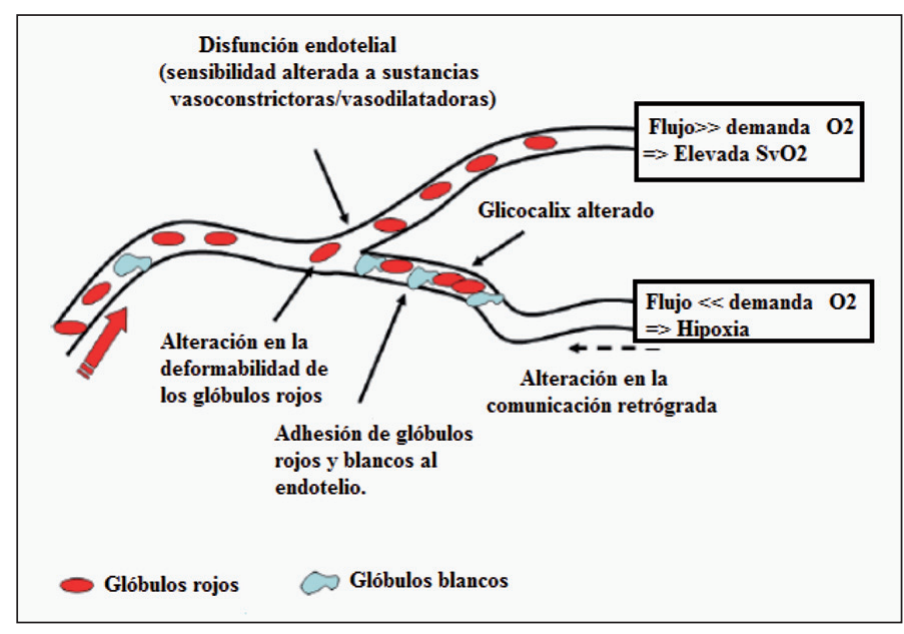

Figura 5. Principales mecanismos implicados en el desarrollo de las alteraciones de la microcirculación. 
tán en un contacto cercano con él. Se describe una disminución en su tamaño y alteración en su composición química, por lo que probablemente juega un rol crucial en el desarrollo de las alteraciones microcirculatorias, como ha sido demostrado en modelos experimentales con infusión de dosis bajas de endotoxina ${ }^{32}$.

\section{¿Son las alteraciones microcirculatorias un evento primario o una respuesta adaptativa a demandas metabólicas alteradas?}

Las alteraciones previamente señaladas deben ser miradas como un evento primario (causal) que origina disfunción celular más que adaptativo (consecuencia de alteraciones metabólicas celulares). La teoría adaptativa se basa en datos que apoyan la existencia de disfunción mitocondrial en sepsis, la cual podría ser responsable de una disminución del flujo sanguíneo microvascular, reflejando así una adaptación a la reducción de las demandas de oxígeno a nivel celular. Sin embargo, existe evidencia contundente que las alteraciones microcirculatorias actúan como un evento primario en los estados inflamatorios, jugando un rol principal en la hipoxia celular y desarrollo del SDOM.

Experimentalmente se ha observado que la disfunción microvascular y el consiguiente desarrollo de falla orgánica se produce debido a la interacción entre las células circulantes y el endotelio, sugiriendo un origen endovascular más que tisular de la disfunción microvascular. En un modelo de ligadura cecal y perforación $^{33}$, se demostró que la extracción de oxígeno a través de un único capilar perfundido de músculo esquelético está aumentada (saturación de oxígeno reducida), lo que indica que la heterogeneidad de la perfusión y no la disfunción celular es la responsable de la capacidad disminuida de extracción de oxígeno en la sepsis, limitando por lo menos en la fase precoz el rol de la hipoxia citopática ${ }^{34}$.

El dato que las alteraciones microcirculatorias están relacionadas a un aumento en la diferencia tejido-arterial de $\mathrm{pCO}_{2}$ es también otra fuerte prueba a favor que estas son un evento primario. En efecto, si el flujo se ajusta a la disminución del metabolismo, la producción tisular de $\mathrm{CO}_{2}$ debiera estar disminuida, no habiendo razón para que aumentara la gradiente tejido-arterial de $\mathrm{pCO}_{2}$, por el contrario, si el flujo es insuficiente en relación a la situación metabólica, entonces el $\mathrm{CO}_{2}$ se acumula a nivel tisular y la diferencia tejido-arterial de $\mathrm{pCO}_{2}$ aumenta ${ }^{35}$.

Por último, los efectos benéficos de la terapia precoz en shock argumentan en contra de un problema de utilización de energía, al menos en los estadios tempranos ${ }^{36}$.

La persistencia de alteraciones microcirculatorias una vez recuperado el shock se asocia con la presencia de disfunciones orgánicas y muerte, lo que ha llevado a plantear que el compromiso de la microcirculación sería el motor del SDOM ${ }^{11}$.

\section{Importancia de la microcirculación y sus alteraciones en sepsis grave}

En condiciones de normalidad la heterogeneidad de la microcirculación es mínima. Como hemos señalado previamente, las alteraciones microcirculatorias se observan en diversas enfermedades además de la sepsis, además de la sepsis. no obstante, estos cambios son más graves en el shock séptico que en otro tipo de shock, a pesar de una similar gravedad en la alteración de la circulación sistémica.

Los efectos fisiopatológicos de estas alteraciones microcirculatorias ${ }^{16}$ son:

1. Heterogeneidad: la coexistencia de unidades capilares bien perfundidas y otras perfundidas intermitente o no perfundidas pudieran ser responsables de la disminución en la capacidad de extracción de oxígeno en los pacientes sépticos (observándose un incremento en la $\mathrm{SvO}_{2}$ ). Es importante destacar que capilares con ausencia de flujo en un momento dado pueden estar bien perfundidos en los siguientes minutos, señalando así que la microcirculación es un proceso dinámico, lo cual se exacerba aún más en pacientes sépticos. Estas alteraciones han sido descritas en diferentes modelos experimentales de sepsis, siendo evaluados en diferentes órganos (piel, lengua, intestino, hígado, riñón, cerebro) y en diversas especies animales. De ahí que estos cambios parecen ser universales y presentar un mecanismo fisiopatológico común. 
Importantemente, la total reversibilidad de las alteraciones microcirculatorias descritas por la aplicación tópica de acetilcolina, sugiere que la completa obstrucción por microtrombos no es un componente esencial de estas alteraciones $^{18}$.

2. Hipoxia tisular: las alteraciones microcirculatorias se asocian con zonas de bajos niveles de $\mathrm{pO}_{2}$ tisular, lo cual no es compatible con alteraciones primarias metabólicas exclusivas.

3. Isquemia/reperfusión: tanto la presencia de flujo intermitente como la ausencia de este en algunos capilares puede llevar a áreas de daño por isquemia/reperfusión y eventualmente a la amplificación de la respuesta inflamatoria y del daño tisular.

En las primeras fases del shock se ha señalado que los indicadores de flujo sanguíneo microcirculatorio pueden servir como marcadores precoces de hipoperfusión tisular y anunciar el inicio de una falla circulatoria y disfunción multiorgánica ${ }^{3}$.

Finalmente, la precocidad de nuestra búsqueda de falla microcirculatoria puede ser trascendente, en base a que el impacto de la disfunción microcirculatoria, por ende, su manipulación, puede ser mayor durante la etapa inicial de resucitación, por lo cual parece tener sustento la hipótesis que la falla orgánica durante la fase aguda de la sepsis es un fenómeno dependiente de perfusión mientras que el desarrollo de falla orgánica en fase tardía, está íntimamente relacionado a disfunción mitocondrial, la cual ocasiona una falla bioenergética.

\section{Conclusiones}

Las alteraciones microcirculatorias son frecuentes de observar en el paciente críticamente enfermo, especialmente en el paciente con sepsis grave-shock séptico.

Estos hallazgos son más graves en los pacientes no sobrevivientes y su persistencia juega un rol preponderante en el desarrollo de disfunción orgánica múltiple y muerte. El reconocimiento precoz de esta disfunción permitiría una resucitación orientada a la normalización de estas alteraciones y así, mejorar la supervivencia de los pacientes sépticos.

\section{Referencias}

1.- Pinsky MR, Payen D: Functional hemodynamic monitoring. Crit Care 2005; 9: 566-72.

2.- Tibby SM, Hatherill M, Marsh MJ, Murdoch IA: Clinicians' abilities to estimate cardiac index in ventilated children and infants. Arch Dis Child 1997; 77: 516-8.

3.- Spronk PE, Zandstra DF, Ince C: Bench-to-bedside review: sepsis is a disease of the microcirculation. Crit Care 2004; 8: 462-8.

4.- Gil Antón J, Cecchetti C, Menéndez S, Cambra FJ, López-Herce J, Rodríguez-Núñez A: Preliminary clinical experience with PiCCO system in children with shock. An Pediatr 2009; 71: 1: 35-40.

5.- López-Herce J, Bustinza A, Sancho L, et al: Cardiac output and blood volume parameters using femoral arterial thermodilution. Pediatr Int 2009; 51: 59-65.

6.- de Oliveira CF, de Oliveira DS, Gottschald AF, et al: ACCM/PALS haemodynamic support guidelines for paediatric septic shock: an outcomes comparison with and without monitoring central venous oxygen saturation. Intensive Care Med 2008; 34: 1065-75.

7.- Bronicki RA: Venous oximetry and the assessment of oxygen transport balance. Pediatr Crit Care Med 2011; 12 (Suppl): S21-6.

8.- Beach JM, McGahren ED, Duling BR: Capillaries and arterioles are electrically coupled in hamster cheek pouch. Am J Physiol 1998; 275: H1489-H1496.

9.- Johnson PC: Autoregulation of blood flow. Circ Res 1986; 59 (5): 483-95.

10.- De Backer, Ortiz JA, Salgado D: Coupling microcirculation to systemic hemodynamics. Curr Opin Crit Care 2010; 16: 250-4.

11.- Ince $C$ : The microcirculation is the motor of sepsis. Critical Care 2005; 9 (Suppl 4): S13-9.

12.- Ellis CG, Jagger J, Sharpe M: The microcirculation as a functional system. Crit Care 2005; 9 (Suppl 4): S3-8.

13.- Abraham E, Singer M: Mechanisms of sepsis-induced organ dysfunction. Crit Care Med 2007; 35: 2408-16.

14.- De Backer D, Dubois MJ: Assessment of the microcirculatory flow in patients in the intensive care unit. Curr Opin Crit Care 2001; 7: 200-3.

15.- De Backer D, Ospina-Tascon G, Salgado D, Favory R, Creteur J, Vincent JL: Monitoring the microcirculation in the critically ill patient: current methods and future approaches. Intensive Care Med 2010; 36: 1813-25.

16.- De Backer D, Hollenberg S, Boerma C, et al: How to evaluate the microcirculation? Report of a round table conference. Critical Care 2007; 11: R101.

17.- Buwalda $M$, Ince $C$ : Opening the microcirculation: can 
vasodilators be useful in sepsis? Intensive Care Med 2002; 28: 1208-17.

18.- De Backer D, Creteur J, Preiser JC, Dubois MJ, Vincent $J L$ : Microvascular blood flow is altered in patients with sepsis. Am J Respir Crit Care Med 2002; 166: 98-104.

19.- Krogh $A$ : The number and distribution of capillaries in muscles with calculations of the oxygen pressure head necessary for supplying the tissue. J Physiol 1919; 52: 409-15.

20.- Pittman RN: Oxygen transport and exchange in the microcirculation. Microcirculation 2005; 12: 59-70.

21.- Collins DM, McCullough WT, Ellsworth ML: Conducted vascular responses: communications across the capillary bed. Microvasc Res 1998; 56: 43-53.

22.- Bolon ML, Peng T, Kidder GM, Tyml K: Lipopolysaccharide plus hypoxia and reoxygenation synergistically reduce electrical coupling between microvascular endothelial cells by dephosphorylating connexin40. J Cell Physiol 2008; 217: 350-9.

23.- Top AP, Van Dijk M, van Velzen JE, Ince C, Tibboel D: Functional capillary density decreases after the first week of life in term neonates. Neonatology 2011; 99: 73-7.

24.- Perera P, Kurban K, Ryan TJ: The development of the cutaneous microvascular system in the newborn. $\mathrm{Br} \mathrm{J}$ Dermatology 1970; 82 (S5): 86-91.

25.- Top AP, Tasker RC, Ince C: The microcirculation of the critically ill pediatric patient. Crit Care 2011; 15: 213.

26.- Boerma EC: The microcirculation as a clinical concept: work in progress. Curr Opin Crit Care 2009; 15: 261-5.

27.- Dietrich HH, Ellsworth ML, Sprague RS, Dacey RG Jr: Red blood cell regulation of microvascular tone through adenosine triphosphate. Am J Physiol Heart Circ Physiol 2000; 278: H1294-8.
28.- Hollenberg SM: Think locally: evaluation of the microcirculation in sepsis. Intensive Care Med 2010; 36: 1807-9.

29.- Tyml K, Wang X, Lidington D, Ouellette Y: Lipopolysaccharide reduces intercellular coupling in vitro and arteriolar conducted response in vivo. Am J Physiol Heart Circ Physiol 2001; 281: H1397-1406.

30.- Piagnerelli M, Boudjeltia KZ, Brohee D, et al: Alterations of red blood cell shape and sialic acid membrane content in septic patients. Crit Care Med 2003; 31: 2156-62.

31.- Croner RS, Hoerer E, Kulu Y, et al: Hepatic platelet and leukocyte adherence during endotoxemia. Crit Care. 2006; 10: R15.

32.- Nieuwdorp M, Meuwese MC, Mooij HL: Tumor necrosis factor-alpha inhibition protects against endotoxin-induced endothelial glycocalyx perturbation. Atherosclerosis 2009; 202 (1): 296-303.

33.- Ellis CG, Bateman RM, Sharpe MD, Sibbald WJ, Gill $R$ : Effect of a maldistribution of microvascular blood flow on capillary $\mathrm{O}(2)$ extraction in sepsis. Am J Physiol Heart Circ Physiol 2002; 282: H156-64.

34.- Goldman D, Bateman RM, Ellis CG: Effect of decreased $\mathrm{O}_{2}$ supply on skeletal muscle oxygenation and $\mathrm{O} 2$ consumption during sepsis: role of heterogeneous capillary spacing and blood flow. Am J Physiol Heart Circ Physiol 2006; 290 (6): H2277-85.

35.- Creteur J, De Backer D, Sakr Y, Koch M, Vincent JL: Sublingual capnometry tracks microcirculatory changes in septic patients. Intensive Care Med 2006, 32: 516-23.

36.- Sakr Y, Dubois MJ, De Backer D, Creteur J, Vincent $J L$ : Persistent microcirculatory alterations are associated with organ failure and death in patients with septic shock. Crit Care Med 2004; 32: 1825-31. 